\title{
Measured energy content of frequently purchased restaurant meals: multi-country cross sectional study
}

\author{
Susan B Roberts, ${ }^{1}$ Sai Krupa Das, ${ }^{1}$ Vivian Marques Miguel Suen, ${ }^{2}$ Jussi Pihlajamäki, ${ }^{3,4}$ \\ Rebecca Kuriyan, ${ }^{5}$ Matilda Steiner-Asiedu, ${ }^{6}$ Amy Taetzsch, ${ }^{1}$ Alex K Anderson, ${ }^{7}$ Rachel E Silver, ${ }^{1,8}$ \\ Kathryn Barger, ${ }^{1}$ Amy Krauss, ${ }^{1}$ Leila Karhunen, ${ }^{3}$ Xueying Zhang, ${ }^{9,10,11}$ Catherine Hambly, ${ }^{11}$ \\ Ursula Schwab, ${ }^{3,12}$ Andresa de Toledo Triffoni-Melo, ${ }^{13}$ Priscila Giacomo Fassini, ${ }^{2}$ \\ Salima F Taylor, ${ }^{1}$ Christina Economos, ${ }^{8}$ Anura V Kurpad, ${ }^{5}$ John R Speakman ${ }^{9,11,14}$
}

For numbered affiliations see end of article.

Correspondence to: SB Roberts

susan.roberts@tufts.edu

(or @JMHNRCA on Twitter;

ORCID 0000-0003-1320-8460) and

J R Speakman

j.speakman@abdn.ac.uk

(or @johnspeakman4 on Twitter)

Additional material is published online only. To view please visit the journal online.

Cite this as: $B M J$ 2018;363:k4864 http://dx.doi.org/10.1136/bmj.k4864

Accepted: 7 November 2018

\section{ABSTRACT}

\section{OBJECTIVE}

To measure the energy content of frequently ordered meals from full service and fast food restaurants in five countries and compare values with US data.

DESIGN

Cross sectional survey.

\section{SETTING}

223 meals from 111 randomly selected full service and fast food restaurants serving popular cuisines in Brazil, China, Finland, Ghana, and India were the primary sampling unit; 10 meals from five worksite canteens were also studied in Finland. The observational unit was frequently ordered meals in selected restaurants.

\section{MAIN OUTCOME MEASURE}

Meal energy content, measured by bomb calorimetry.

RESULTS

Compared with the US, weighted mean energy of restaurant meals was lower only in China (719 (95\% confidence interval 646 to 799 ) $\mathrm{kcal}$ versus 1088 (1002 to 1181) kcal; P<0.001). In analysis of variance models, fast food contained $33 \%$ less energy than ful service meals ( $\mathrm{P}<0.001)$. In Finland, worksite canteens provided $25 \%$ less energy than full service and fast food restaurants (mean 880 (SD 156) versus 1166 (298); $P=0.009)$. Country, restaurant type, number of meal components, and meal weight predicted meal energy in a factorial analysis of variance $\left(R^{2}=0.62\right.$, $\mathrm{P}<0.001)$. Ninety four per cent of full service meals and $72 \%$ of fast food meals contained at least $600 \mathrm{kcal}$.

\section{WHAT IS ALREADY KNOWN ON THIS TOPIC}

The prevalence of obesity continues to increase in most countries

Fast food has been proposed as an important contributor to global obesity on the basis of nutritional information reported by large chain restaurants

Little is known about other types of restaurant meals owing to a lack of data on measured meal energy content

\section{WHAT THIS STUDY ADDS}

Of five countries (Brazil, China, Finland, Ghana, and India), only restaurants in China served meals containing significantly less energy than restaurants in the US In contrast to widespread assumptions, fast food meals contained $33 \%$ less dietary energy on average than meals from full service restaurants

The high energy content of restaurant meals may be an important contributor to the global obesity epidemic and a potentially impactful target for public health interventions

Modeling indicated that, except in China, consuming current servings of a full service and a fast food meal daily would supply between $70 \%$ and $120 \%$ of the daily energy requirements for a sedentary woman, without additional meals, drinks, snacks, appetizers, or desserts.

\section{CONCLUSION}

Very high dietary energy content of both full service and fast food restaurant meals is a widespread phenomenon that is probably supporting global obesity and provides a valid intervention target.

\section{Introduction}

The prevalence of obesity is at epidemic levels in most countries. ${ }^{12}$ In additionto being the underlying cause of most non-communicable diseases, ${ }^{1}$ obesity is a major risk factor for dementia, Alzheimer's disease, and disability in old age, ${ }^{34}$ and it challenges national economies through its detrimental effects on workforce productivity, healthcare costs, and rates of disability. ${ }^{5-9}$ The World Health Organization and many national governments have issued calls to action to reduce population obesity. ${ }^{10}$ However, the prevalence of obesity continues to rise, showing that effective interventions have yet to be identified.

The increase in per capita dietary energy worldwide, especially from refined carbohydrates and fats, ${ }^{11}$ identifies increased energy intake as an important facilitator of global obesity. However, the development of effective interventions to reduce overeating requires identification of specific eating practices, environmental factors, or both that are quantitatively important and malleable. Fast food has been widely implicated as one such target on the basis of its high energy content and a variety of research findings including a positive association between number of fast food restaurant transactions and rates of obesity across countries. ${ }^{12} 13$ However, studies of the spatial association of the density of fast food outlets and rates of obesity provide conflicting evidence. ${ }^{14-16}$ Furthermore, the prevalence of obesity increased to a greater extent in a region of the US with zoning interventions to reduce access to fast food, ${ }^{17}$ although this single study finding needs to be replicated. Nevertheless, these data call into question the presumed role of fast food in the obesity epidemic.

A major limitation of previous research on restaurants and obesity is that almost all studies examining the composition of meals have used nutritional 
data provided by large chain restaurants. ${ }^{12} 1819$ This approach excludes restaurants that do not provide nutritional information, which is the case for most full service and local fast food restaurants. The limited information available on measured energy contents indicates that restaurants in general, rather than fast food restaurants specifically, provide very high energy levels. In some cases, restaurant meals supply a whole day's energy needs in a single entrée. ${ }^{20-24}$ Furthermore, the positive relation between frequency of eating out and higher overall energy intake and weight gain is consistent with experimental data suggesting a low ability to compensate for bouts of overeating by equivalent undereating on other occasions. ${ }^{25-29}$ However, almost all data have been collected in the US, ${ }^{20} 2123$ and very little information is available from other countries, ${ }^{24}$ especially the low and middle income countries where rates of obesity are rising very rapidly.

This study measured the energy contents of the most frequently ordered meals in full service and fast food restaurants in five countries (Brazil, China, Finland, Ghana, and India), as well in worksite canteens in Finland. We compared data with the measured energy contents of the most frequently ordered US restaurant meals to test the hypothesis that large restaurant portions are not a phenomenon unique to the US and to identify other countries where portion sizes may be problematic.

\section{Methods}

This study measured the energy content of a representative sample of 223 popular meals purchased from 111 randomly selected full service (sit down meal served by waitperson and eaten in premises) and fast food (counter served meal eaten on or off premises) restaurants in a major city in each of six countries (Ribeirao Preto in Brazil, Beijing in China, Kuopio in Finland, Accra in Ghana, Bangalore in India, and Boston in the US). In addition to the restaurant orders, 10 meals were obtained from five worksite canteens in Finland as an exploratory study component to compare data with regular full service and fast food restaurants. We included this last evaluation because worksite canteens in Finland typically offer subsidized lunches to support employees' health and are included in national dietary recommendations. ${ }^{30}$ We collected and analyzed meals for Brazil, China, Ghana, Finland, and India between 2014 and 2017. We compared data with comparable information for the most 100 popular meal choices in US restaurants. We analyzed a total of 233 meals including the US sample and the Finland canteens.

We selected countries from diverse regions of the world to provide data, and we chose the specific sites because they had experienced researchers able to implement the local protocol. We chose the US as the comparison site because of existing comparable data on the most popular US menu orders. We extracted the US data from two previous studies that also randomly selected restaurants and measured the most frequently ordered meals, ${ }^{20} 23$ with three additional randomly selected fast food restaurants for comparability across sites. Full service restaurants in the US data included the most popular ethnic choices (American, Mexican, Chinese, Italian, and Japanese), reflecting the multiethnic nature of popular US cuisines.

For the purpose of this study, we defined a "meal" as the main course with side dishes that were included at no extra charge. We did not include drinks, appetizers, and desserts that required extra payment. We used this approach because the focus of the study was to compare the most commonly ordered meals across countries, recognizing that we likely underestimated total energy content of typical meal orders owing to the non-inclusion of drinks and perhaps additional courses.

\section{Selection of restaurants and meals}

The restaurant selection process involved local research teams using internet searches and site visits to identify all full service and fast food establishments in a predefined geographic area of approximately $25 \mathrm{~km}^{2}$ in the locality of the research center in each country. Buffet restaurants and hot pot restaurants (which are equivalent to buffets in terms of self selected portion sizes) were not included. Each restaurant was then categorized by ethnicity (local, international, specific other national), with the goal of excluding international and specific national restaurants from the selection process unless they were widespread (defined as $>15 \%$ of total restaurants). As restaurants in Ghana are used for both full service meals and fast food, selected eating establishments at this site were not distinguished by restaurant type.

Full service and fast food restaurants were randomly selected for inclusion in the study by use of a random number generator in SAS 9.4, and back-up sites were also randomly identified for use if initial selections were closed or ineligible or failed to have the meals previously identified for collection at the time the order was placed. Restaurants are not identified by name in the data presentations because they were selected randomly to represent general restaurant categories. The local research staff called each selected restaurant and asked to speak with the restaurant manager to obtain information on the most frequently ordered main course meals. These calls were made before the selected meals were ordered for collection and analysis. We chose this method of selecting the most frequently ordered meals because we found no national or regional statistics to determine the most frequent orders at each site.

\section{Meal collection and energy determination}

Local research staff posed as restaurant patrons and ordered the target meals by following a standard procedure. Orders were placed for the identified meals in person at the time of the main meal in that country. Collected foods included all those that came as part of the meal order but did not include any additional items that required further payment. Any drinks that 
came with the meal were excluded from analyses. The researcher requested the meal to take away with all items packed separately.

The two most popular meals in each randomly selected restaurant were targeted for collection in Brazil, Ghana, Finland, and India (five full service and five fast food restaurants per country). In addition, the 10 most popular meals from five worksite canteens in Finland were included as an exploratory study component. As the work in China was part of a larger investigation, and restaurant goers in full service restaurants in China routinely share individual items among people dining together, a different method of sample collection was necessary. A single full service meal order was placed for the five (four at one location) most popular items in each restaurant ( 74 foods items across 15 full service restaurants), and information was divided by the number of items to yield an energy equivalent meal size for a single diner. This method approximates the traditional practice of consuming an average of one item per person, with a greater number of items ordered for variety and so that leftovers can be taken home.

After the purchase, meals were taken to the laboratory and individual components were separated from each other if not previously done by the restaurant. Total meals and meal components were then weighed to within $0.1 \mathrm{~g}$ on a calibrated electronic balance before preparation for analysis by bomb calorimetry. Samples from Brazil, Ghana, Finland, and India were shipped to Tufts University for analysis because the sites had no local bomb calorimeter, whereas samples from China were analyzed by the local investigator's team. Samples were prepared as previously described. ${ }^{22}$ Briefly, the meals were homogenized to a smooth puree, freeze dried to constant weight, and reground in a food processor to a fine, dry powder. The food powders were then analyzed for gross energy content in duplicate by bomb calorimetry (Parr 1261 at Tufts University and Parr 6100 with a 1109 semi-micro bomb for China samples; Parr Instruments, Moline, IL, USA). Both bomb calorimeters were standardized daily with benzoic acid pellets, and all calibration values were within $1 \%$ of the known heat of combustion for benzoic acid. Total energy content was determined for each meal as the product of total dried food weight and the mean heat of combustion of the duplicate dry samples. Energy density was calculated as gross energy per gram of fresh meal weight. In addition, we calculated the percentage of meals containing at least 600 and at least $2000 \mathrm{kcal}$ metabolizable energy, with adjustment for the obligatory approximate $10 \%$ energy losses in urinary nitrogenous compounds and fecal energy. ${ }^{3132}$ We did the $600 \mathrm{kcal} / \mathrm{meal}$ calculation because this is a benchmark that has recently been adopted as a public health recommendation for a healthy level of meal energy in the UK. ${ }^{33}$ We did the $2000 \mathrm{kcal} / \mathrm{meal}$ calculation because this is the total daily energy requirement of a typical woman.

\section{Statistical analysis}

We compared the mean energy content of meals (primary outcome) for Brazil, China, Ghana, Finland, and India with the US data. A retrospective power calculation indicated that the sample size was sufficient to detect an approximate $180 \mathrm{kcal} / \mathrm{meal}$ difference between the US and the international sites with $80 \%$ power. We also examined meal weight $(\mathrm{g})$ and energy density ( $\mathrm{kcal} / \mathrm{g})$. We obtained descriptive statistics for each measurement for each country by restaurant type (full service and fast food). We compared full service and fast food meals within each country by using an independent samples $t$ test. We calculated sample weighted mean energy content, meal weight, and energy density separately for each country on the basis of the number of measured full service and fast food meals. All measurements were natural-log transformed for use in statistical tests to meet model assumptions for normality. We compared weighted mean energy content, meal weight, and energy density for each county with the US by using an analysis of variance model. We used factorial analysis of variance to evaluate the major predictors of meal energy content, including country, restaurant type, and number of individual foods per meal. We assessed effect modification of country by both restaurant type and number of foods. We calculated least squares means and 95\% confidence intervals. We compared worksite canteen meals with the combined full service and fast food restaurants by using the Wilcoxon rank sum test. We used SAS 9.4 for all statistical analyses and considered a P value below 0.05 to be statistically significant. In addition to statistical analysis of the measured data, modeling of the impact of the results is given in the supplementary appendix together with a description of how the modeling was conducted, as outlined below.

\section{Patient and public involvement}

No patients or members of the public were directly involved in this study, other than as employees of restaurants that were targeted for covert meal collection. There are no plans to involve patients or the public in the dissemination of results.

\section{Results}

We found a significant positive association between meal weight and meal energy content $\left(R^{2}=0.53\right.$; $\mathrm{P}<0.001$; fig 1), although the moderate strength of the correlation indicates that meal weight by itself was a very imprecise predictor of meal energy content. In addition, a significant, negative association existed between meal energy density and meal weight $\left(\mathrm{R}^{2}=0.10 ; \mathrm{P}<0.001\right.$; fig 1$)$. The descriptions and energy contents of individual meals for each restaurant are given in supplementary table $\mathrm{A}$.

The total energy content of fast food meals was significantly lower than that of meals from full service restaurants in Brazil (34\%; $\mathrm{P}=0.03)$, China (46\%; $\mathrm{P}<0.001)$, and the US (29\%; $\mathrm{P}<0.001)$ (table 1$)$. 


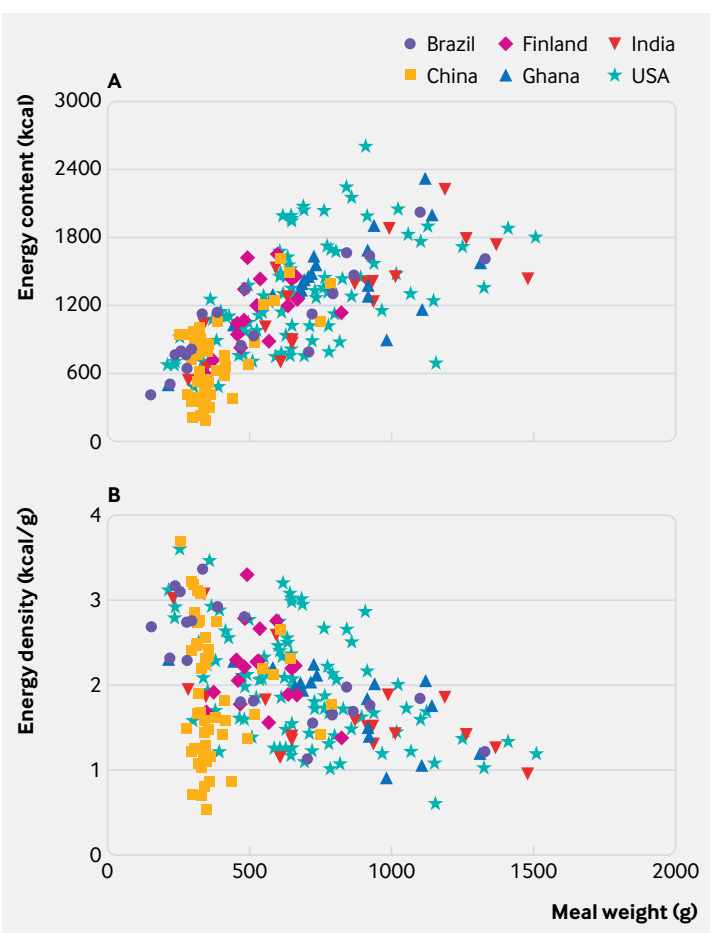

Fig 1 | Scatterplot of associations of meal weight with energy content $\left(R^{2}=0.53 ; P<0.001\right)$ and energy density $\left(R^{2}=0.10 ; P<0.001\right)$ for popular meals served in full service and fast food restaurants in six countries

Weights of fast food portions in these countries were also significantly lower than for meals from full service restaurants $(\mathrm{P}=0.002, \mathrm{P}<0.001$, and $\mathrm{P}<0.001$ for Brazil, China, and the US, respectively), whereas the energy density (kcal/g) of fast food meals was higher in Brazil $(\mathrm{P}=0.003)$ and lower in China $(\mathrm{P}=0.005)$ than the energy density of meals from full service restaurants.
Only China had fast food meals that had less energy than US fast food (by 44\%; $\mathrm{P}<0.001$ ), and similarly only China had full service restaurant meals that had less energy than full service meals in the US (by 21\%; $\mathrm{P}=0.009$ ). The unadjusted mean energy content for all countries was 1317 (SD 442) kcal for full service meals and 809 (363) kcal for fast food meals (table 1). In addition, when we used country weighed values, $94 \%$ of meals from full service restaurants and $72 \%$ of fast food meals across all sites contained at least 600 metabolizable kcal, and 3\% of meals from full service restaurants (including meals from Ghana, India, and the US) contained at least $2000 \mathrm{kcal} /$ serving.

Figure 2 shows the mean differences in meal energy content, meal weights, and meal energy density across countries, using weighted mean values that incorporate the sample sizes for different meal types. In the primary study analysis, a comparison of meal energy content from Brazil, China, Ghana, Finland, and India with US data, only China had meal energy contents that were significantly less than the US (719 (95\% confidence interval 646 to 799) kcal versus 1088 (1002 to 1181) kcal). In addition, only China had lower meal weights than US values (392 (353 to 435) g versus 562 (518 to 610) g) and also lower meal energy density (1.8 (1.6 to 2.0) $\mathrm{kcal} / \mathrm{g}$ versus 1.9 (1.8 to 2.1 ) $\mathrm{kcal} / \mathrm{g}$ ). We did not identify any other significant comparisons.

Table 2 shows the multiple variable predictors of meal energy content. In model I $\left(\mathrm{R}^{2}=0.44\right.$; $\left.\mathrm{P}<0.001\right)$, which included country and restaurant type, we observed a strong effect of country on meal energy content, and post hoc testing indicated that China had significantly lower meal energy content compared with US values (39\%; $\mathrm{P}<0.001)$. Restaurant type also had a significant effect, and meals from fast food restaurants had lower energy contents than meals from full service restaurants, by $33 \%$ on average $(\mathrm{P}<0.001)$.

\begin{tabular}{|c|c|c|c|c|c|c|c|}
\hline Country and restaurant type & No of meals & Energy content (kcal) & $P$ value* & Weight (g) & $P$ value* & Energy density (kcal/g) & $P$ value* \\
\hline \multicolumn{8}{|l|}{ Brazil: } \\
\hline Full service & 10 & $1301(453)$ & \multirow[t]{2}{*}{0.03} & $780(327)$ & \multirow[t]{2}{*}{0.002} & $1.82(0.56)$ & \multirow[t]{2}{*}{0.003} \\
\hline Fast food & 10 & $862(285)$ & & $337(122)$ & & $2.63(0.52)$ & \\
\hline \multicolumn{8}{|l|}{ China: } \\
\hline Full service & 15 & $1045(289)$ & \multirow[t]{2}{*}{$<0.001$} & $484(169)$ & \multirow[t]{2}{*}{$<0.001$} & $2.32(0.73)$ & \multirow[t]{2}{*}{0.005} \\
\hline Fast food & 40 & $561(236)$ & & $338(30)$ & & $1.67(0.71)$ & \\
\hline \multicolumn{8}{|l|}{ Finland: } \\
\hline Full service & 10 & $1135(346)$ & \multirow[t]{2}{*}{0.48} & $500(102)$ & \multirow[t]{2}{*}{0.08} & $2.25(0.52)$ & \multirow[t]{2}{*}{0.45} \\
\hline Fast food & 8 & $1205(242)$ & & $593(117)$ & & $2.06(0.45)$ & \\
\hline Worksite & 10 & $880(156)$ & - & $668(93)$ & - & $1.34(0.27)$ & - \\
\hline \multicolumn{8}{|l|}{ Ghana: } \\
\hline Full service/fast food & 20 & $1412(392)$ & - & $815(256)$ & - & $1.82(0.41)$ & - \\
\hline \multicolumn{8}{|l|}{ India: } \\
\hline Full service & 10 & $1414(488)$ & \multirow[t]{2}{*}{0.22} & $855(416)$ & \multirow[t]{2}{*}{0.59} & $1.86(0.63)$ & \multirow[t]{2}{*}{0.54} \\
\hline Fast food & 10 & 1129 (389) & & $731(311)$ & & $1.68(0.53)$ & \\
\hline \multicolumn{8}{|l|}{ USA: } \\
\hline Full service & 71 & $1362(469)$ & \multirow[t]{2}{*}{$<0.001$} & $741(266)$ & \multirow[t]{2}{*}{$<0.001$} & $1.96(0.65)$ & \multirow[t]{2}{*}{0.27} \\
\hline Fast food & 19 & $969(283)$ & & $475(134)$ & & $2.12(0.66)$ & \\
\hline \multicolumn{8}{|l|}{ All countries: } \\
\hline Full service & 136 & $1317(442)$ & - & 717 (285) & - & $1.98(0.63)$ & - \\
\hline Fast food & 87 & 809 (363) & - & $436(188)$ & - & $1.92(0.71)$ & - \\
\hline
\end{tabular}



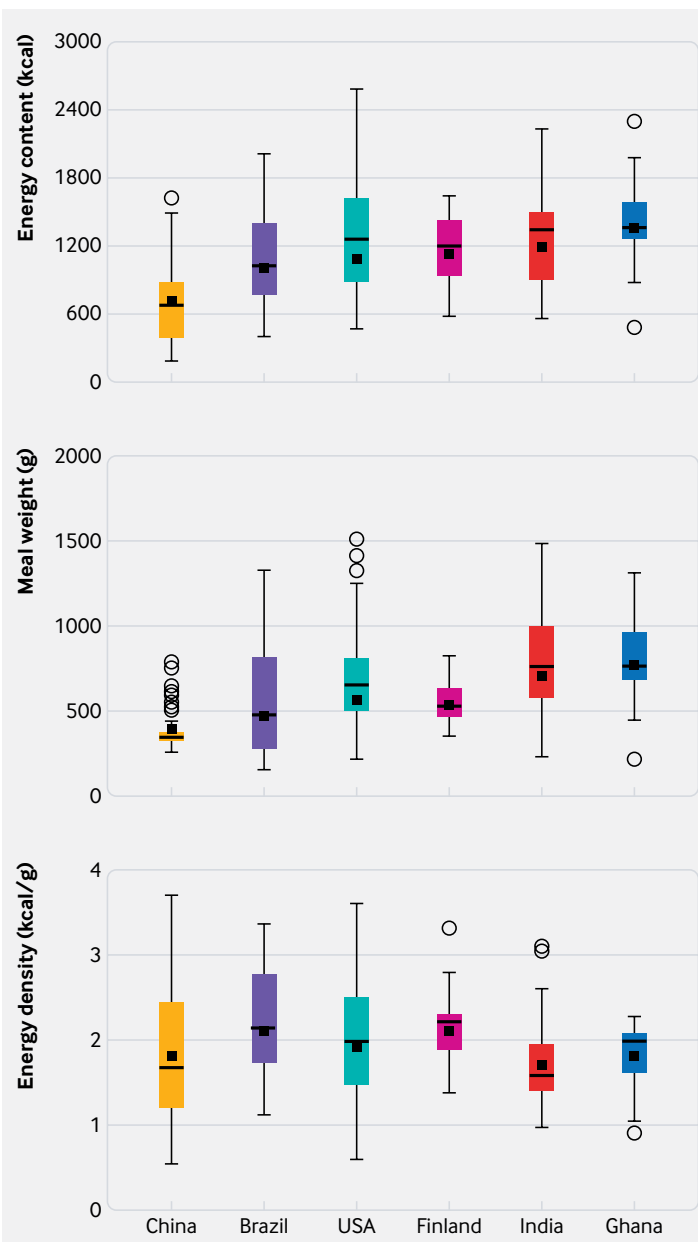

Fig 2 | Energy content, weight, and energy density of restaurant meals in six countries. Boxplots show unweighted fifths (five number summary) and weighted means (indicated by filled square)

Model II shows the prediction of meal energy content by country, restaurant type, and an additional independent variable for the number of food items within the meal $\left(\mathrm{R}^{2}=0.53 ; \mathrm{P}<0.001\right)$. Country of origin $(\mathrm{P}<0.001)$, restaurant type $(\mathrm{P}=0.003)$, and number of food items per meal $(\mathrm{P}<0.001)$ were all significant variables in this model. Model III shows the best fitting model $\left(\mathrm{R}^{2}=0.62 ; \mathrm{P}<0.001\right)$, which predicted meal energy content from country, restaurant type, number of foods, and meal weight. The significantly lower energy content of meals from China compared with the US was retained in this model. Number of foods and meal weight were also significant, but type of meal (full service versus fast food) was not. These analyses show that only China had meals that were lower in energy content than the US, that fast food meals had less energy than full service meals, and that the lower energy content of fast food was in part explained by smaller portion sizes in the meal. In addition, the modeling of the impact of meal energy provided in the supplementary appendix (supplementary tables B-H and supplementary figures $A-G$ ) indicated that, except in China, consuming current servings of a full service and a fast food meal daily would supply between $70 \%$ and $120 \%$ of the daily energy requirements of a sedentary woman (depending on the country), without additional meals and snacks and without the drinks, appetizers, and desserts that were excluded from the definition of a meal in this study.

To examine meal energy content and other meal variables in worksite canteens in Finland, we compared values with combined data from full service and fast food restaurants (fig 3). Canteen meals had a significantly lower mean energy content, by $25 \%$ on average, compared with the full service and fast food meals (mean 880 (156) v 1166 (298) kcal; P=0.009). In addition, the canteen meals had higher meal weight and lower energy density $(\mathrm{P}=0.004$ and $\mathrm{P}<0.001$, respectively).

\section{Discussion}

The increasing global per capita energy intake, especially from refined carbohydrates and fats, ${ }^{11}$ as

\begin{tabular}{|c|c|c|c|c|c|c|}
\hline \multirow[b]{2}{*}{ Variable } & \multicolumn{2}{|l|}{ Model I } & \multicolumn{2}{|l|}{ Model II } & \multicolumn{2}{|l|}{ Model III } \\
\hline & Coefficient $(95 \% \mathrm{Cl})$ & $P$ value & Coefficient $(95 \% \mathrm{Cl})$ & $P$ value & Coefficient $(95 \% \mathrm{Cl})$ & $P$ value \\
\hline \multicolumn{7}{|l|}{ Country of origin: } \\
\hline Brazil* $^{*}$ & $0.93(0.77$ to 1.13$)$ & 0.45 & $0.93(0.79$ to 1.12$)$ & 0.44 & $1.02(0.87$ to 1.20$)$ & 0.79 \\
\hline China* & $0.61(0.53$ to 0.71$)$ & $<0.001$ & $0.70(0.59$ to 0.82$)$ & $<0.001$ & $0.81(0.70$ to 0.93$)$ & 0.005 \\
\hline Finland* & $1.00(0.81$ to 1.23$)$ & 0.99 & $0.91(0.76$ to 1.12$)$ & 0.40 & 1.03 (0.86 to 1.23$)$ & 0.72 \\
\hline India* & $1.04(0.82$ to 1.32$)$ & 0.72 & $0.95(0.76$ to 1.20$)$ & 0.67 & $0.98(0.80$ to 1.20$)$ & 0.84 \\
\hline United States & Referent & - & Referent & - & Referent & - \\
\hline \multicolumn{7}{|l|}{ Restaurant type: } \\
\hline Fast food & $0.66(0.56$ to 0.78$)$ & $<0.001$ & $0.78(0.66$ to 0.90$)$ & 0.003 & 0.91 (0.78 to 1.06) & 0.22 \\
\hline Full service & Referent & - & Referent & - & Referent & - \\
\hline \multicolumn{7}{|l|}{ No of foods per meal: } \\
\hline More than one & - & - & $1.35(1.16$ to 1.57$)$ & $<0.001$ & 1.21 (1.06 to 1.39$)$ & 0.006 \\
\hline One & - & - & Referent & - & Referent & - \\
\hline Meal weight (g) & - & - & - & - & 1.58 (1.39 to 1.80$)$ & $<0.001$ \\
\hline Country $\times$ restaurant type & $1.03(0.96$ to 1.11$)$ & 0.43 & $1.04(0.97$ to 1.11$)$ & 0.30 & 0.99 (0.93 to 1.05) & 0.72 \\
\hline Country $\times$ food number & - & - & $0.93(0.85$ to 1.02$)$ & 0.12 & 0.97 (0.90 to 1.05$)$ & 0.39 \\
\hline Model $R^{2}$ & 0.44 & $<0.001$ & 0.53 & $<0.001$ & 0.62 & $<0.001$ \\
\hline
\end{tabular}



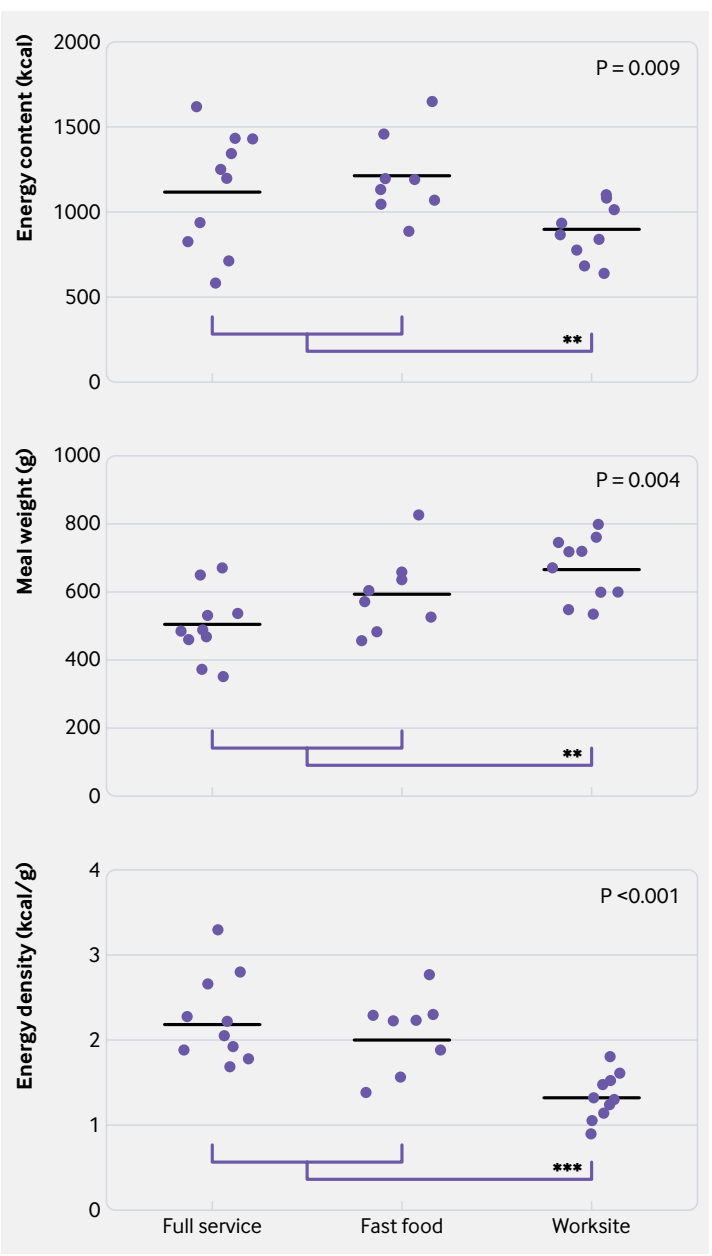

Fig 3 | Individual and mean values for energy content, meal weight, and energy density of meals served in different restaurant types in Finland. Canteen values differed significantly from weighted values for full service and fast food restaurants. ${ }^{* \star P} \mathrm{P}<0.01$. ${ }^{\star \star \star} \mathrm{P}<0.001$

well as the positive association between rising rates of obesity and increasing energy intake across different countries, ${ }^{34}$ highlights the central role that overeating has played in the global obesity epidemic. However, identifying specific aspects of eating behavior, the food environment, or both that are quantitatively important and can lead to effective public health interventions has been challenging. To our knowledge, this is the first study of measured restaurant meal energy contents across different countries, allowing us to examine meals from full service and local fast food restaurants that do not routinely provide nutritional information. Notably, given the previous focus on high meal energy content as a particular problem in the US, only one country (China) had an average meal energy content that was significantly lower than the US value. In addition, substantial variability existed in meal energy content within countries, and in some cases more than a twofold difference existed in the energy content of the same meals purchased in different restaurants (for example, the same meal in China had 1386 and $657 \mathrm{kcal}$ in restaurants 37 and 39 in supplementary table A), which would make providing consumers with guidance on how to eat less in restaurants extremely challenging in the absence of uniform menu labeling. Nevertheless, we observed some patterns-for example, single orders of popular meals ordered in fast food restaurants contained 33\% less energy than meals in full service restaurants, in direct contrast to previous suggestions that the problem of excessive meal energy contents is specific to fast food. These results are broadly consistent with previous work by our team in the US in identifying restaurant meals in general, rather than fast food specifically, as an important target for interventions to reduce obesity, ${ }^{20-23}$ and they show that this is a global rather than a specifically US problem.

\section{Strengths and limitations of study}

Implementing comparable protocols across six countries is challenging, and the study has several limitations. Firstly, despite the very large measured energy content of restaurant meals, this study almost certainly underestimates the restaurant meal orders made by consumers, because we analyzed only main course meals (entrées plus side dishes provided with them), whereas people dining out will typically order drinks, and sometimes appetizers and desserts, as well. In addition, although we collected the most popular orders as defined by the restaurants, we had no independent data on the frequency of meal purchases. Some restaurant patrons may also consume multiple meals at the same sitting, which would have resulted in further underestimation of meal energy. Seasonality was also not considered, and sampling was restricted to a specific urban area in each country. Concerning China specifically, because full service meals are consumed communally, the assumptions made in sample collection (an equal number of food items and diners on average) necessarily influenced the accuracy of projected meal energy levels. An additional general limitation was that most of the reference US data were collected about three years earlier than the data for other countries, but this is unlikely to have had a more than a small effect. We also assumed that the size of meals collected for taking away was the same as those supplied to diners inside the restaurants. These limitations notwithstanding, this study provides the first multinational data on measured energy contents of popular meals from full service and fast food restaurants and, as such, provides unique information relevant to the global obesity epidemic.

\section{Interpretation and policy implications}

Very high energy content of restaurant meals is problematic for several reasons. In particular, the frequency of consuming meals prepared away from home is increasing worldwide. ${ }^{35-38}$ In addition, even the average portion sizes measured in this study are very high relative to human energy requirements, especially for people with lower than average requirements such as sedentary people, older women, and people below average height. Our supplementary modeling data show that two average sized restaurant 
meals a day (one from a fast food restaurant and one in a full service restaurant) would provide almost all the daily energy requirement of a person with one standard deviation lower energy requirements than average, without any additional meals, drinks, snacks, appetizers, or desserts.

Large restaurant meals as measured here would not necessarily be a problem if people ate them infrequently, but, as noted above, dining out is now common. In addition, three meals and one or more snacks daily is usual worldwide, including in the countries studied here. ${ }^{39}$ In addition, even the average ${ }^{40-45}$ Excessively sized restaurant meals may also normalize overeating in other situations, ${ }^{46}$ with a resulting further increase in energy intake. Although people eating out frequently may try to compensate by choosing smaller meals, the consistent associations between frequent eating out and body mass index suggest that this strategy is, at the very least, not fully effective. ${ }^{25-28}$ Part of the explanation for this phenomenon seems to be that humans have a poor ability to compensate for overeating at one meal by eating less at other meals, especially for higher energy meals and liquid calorie sources, ${ }^{47}$ resulting in a net increase in energy intake when eating of restaurant meals is frequent. ${ }^{25} 27$ This may be due in part to the cephalic phase initiation of digestion, which is a normal physiologic mechanism whereby the sight and smell of food causes parasympathetic mediated preparations for digestion that include increased hunger, enlarged stomach lumen, and greater gastric motility. ${ }^{484}$ Effectively, the human body is in equilibration with the immediate food environment via its cephalic phase, with the result that human eating patterns tend to synchronize with the available amount of food, whatever that amount is. Another innate mechanism that may contribute to overeating when portion sizes are very large is an increase in bite size without a compensatory decrease in the rate of bites, which results in a faster rate of energy ingestion. ${ }^{50}$

The results of this study support menu labeling for all restaurant foods, because in studies of large chain fast food restaurants, the introduction of labeling has been associated with reduced energy content of offered menu items over time, ${ }^{51}$ and the same benefit could potentially be achieved in all restaurants with uniform labeling requirements. We have previously provided an open access web tool to support recipe calculation by restaurants. ${ }^{52}$ In addition, our results suggest that recent public health recommendations to reduce restaurant meal servings to $600 \mathrm{kcal}$ could become an important contributor to reducing the prevalence of obesity worldwide, by helping to prevent weight gain and providing active support for weight loss. ${ }^{33}$ Results of our supplementary modeling indicate that if restaurant meals were reduced to 600 kcals, even US adults with low energy requirements could consume up to seven restaurant meals a week without consuming more than $20 \%$ of daily requirements on average. However, 94\% of meals from full service restaurants and $74 \%$ of meals from fast food restaurants contained more than $600 \mathrm{kcal}$ in this study, indicating that substantial changes would be needed in all countries if the new guidelines were broadly adopted. We have also previously suggested that allowing restaurants to serve any meal size, as long as they additionally offer physiologically reasonable serving sizes of the same meals at a proportionally reduced price, could be a flexible option that would provide consumers with a strong economic incentive to reduce overeating when eating out. ${ }^{21}$ This option would also take into account the fact that many but not all people would benefit from meals as small as 600 kcal, allowing for larger orders to be placed by those who want a larger portion. Focus group studies have indicated that restaurant owners see ways to make proportional pricing mechanisms feasible, including by offsetting the lower profit margin on smaller portions by increasing the price of larger portions. ${ }^{53}$

During the past decade, substantial attention has been given to the potential role of fast food in the global spread of obesity, and public health interventions to reduce fast food consumption have been promoted. ${ }^{13}$ However, one intervention that reduced the availability of fast food with zoning regulations was associated with an increase in the prevalence of obesity rather than the reverse, ${ }^{17}$ and studies of the spatial association of obesity and fast food restaurants provide conflicting results. ${ }^{14-16}$ Our study found that single servings of fast food meals contained less-not more-energy on average than full service meals (unweighted means: 809 v $1317 \mathrm{kcal} / \mathrm{meal}$ ), and significantly lower values were also seen in three countries (Brazil, China, and US). It is also noteworthy that, although most large fast food chains did not meet the predefined criteria for restaurant density to be included in this study, local fast food restaurants are very common worldwide and have been shown to serve meals with energy contents that were broadly comparable. ${ }^{12} 1854$ Overall, the results of this study suggest that the controversy about the importance of fast food in the global obesity epidemic may be due to the fact that international fast food is only one of several types of meals prepared away from home that are served in excessive portion sizes. Our findings further suggest that new public health interventions should consider all types of meals prepared away from home as targets for intervention, not just meals from fast food restaurants providing nutritional information.

Two countries among the ones tested stand out as having potentially positive lessons for future interventions. Although, as noted above, there is greater uncertainty in the accuracy of the data from China than from the other countries in the study, China had 34\% lower mean meal energy contents than the US (weighted means: $719 v 1088 \mathrm{kcal} / \mathrm{meal}$ ) and the lowest mean fast food meal energy content of any country, which is likely to be a far greater difference than can be accounted for by the possible bias in sample collections. The number of countries tested was too small to analyze the relation of the energy content of restaurant meals with the prevalence of obesity, but nevertheless it is noteworthy that China has the 
lowest prevalence of obesity of any country in this study. ${ }^{1}$ Finland was another notable country because, although the energy contents of commercial full service and fast food restaurants were not different from US values, a finding consistent the high prevalence of obesity nationwide, ${ }^{55}$ the worksite canteen meals contained $25 \%$ less energy than full service and fast food restaurants (880 $\vee 1166 \mathrm{kcal} / \mathrm{meal})$. Worksite canteens in Finland are supported by social policies and included in dietary recommendations, and their widespread use has been linked to improved dietary habits. ${ }^{56}$

In contrast to the positive examples of China and Finland, Ghana and India had restaurant meal energy contents that were not different from the high values observed in the US, even though they are lower-middle income countries with limited financial resources for the treatment of diabetes, cardiovascular disease, and other non-communicable diseases resulting from obesity. Little information is available from these countries on the frequency of consumption of restaurant meals, but high meal energy content is likely to have a particularly negative impact, because smaller body sizes mean that restaurant meals are disproportionately large relative to energy requirements. As in the US, trends in these countries seem to be driven by a combination of consumer demand and the profits being made by food service industries, ${ }^{57} 58$ indicating that government led interventions are more likely to be effective than voluntary industry sponsored changes.

\section{Conclusions and future research}

This study found that the measured energy content of meals from restaurants in Brazil, Ghana, India, and Finland were not significantly different from the very high values previously reported for the US, and only one country (China) had measured values lower than the US. In addition, and contrary to widespread assumptions, fast food meals contained significantly less energy than did meals from full service restaurants. Further studies are needed to expand testing of restaurants in different countries and locations. Nevertheless, the consistency of results obtained here across very diverse countries suggests that the very high energy content of meals prepared away from home generally, rather than of fast food specifically, may be an important contributor to the global obesity epidemic and a potentially impactful target for public health interventions.

\section{AUTHOR AFFILIATIONS}

${ }^{1}$ Jean Mayer USDA Human Nutrition Research Center on Aging, Tufts University, Boston, MA 02111, USA

${ }^{2}$ Ribeirão Preto Medical School of University of Sao Paulo, Ribeirao Preto, SP, 14049 900, Brazil

${ }^{3}$ Institute of Public Health and Clinical Nutrition, School of Medicine, University of Eastern Finland, Kuopio, 70211, Finland

${ }^{4}$ Clinical Nutrition and Obesity Center, Kuopio University Hospital, Kuopio, 70211, Finland

${ }^{5}$ Division of Nutrition, St John's Research Institute, Bengaluru, 560034, India

${ }^{6}$ Department of Nutrition and Food Science, University of Ghana, Legon-Accra, Ghana
${ }^{7}$ Department of Foods and Nutrition, University of Georgia, Athens, GA, USA

${ }^{8}$ Friedman School of Nutrition Science and Policy, Tufts University, Boston, MA 02111, USA

${ }^{9}$ State Key Laboratory of Molecular Developmental Biology, Institute of Genetics and Developmental Biology, Chinese Academy of Sciences, Beijing 100101, PRC

${ }^{10}$ Chinese Academy of Sciences, Shijingshan District, Beijing 100049, PRC

${ }^{11}$ Institute of Biological and Environmental Sciences, University of Aberdeen, Aberdeen AB24 2TZ, UK

${ }^{12}$ Department of Medicine, Endocrinology and Clinical Nutrition, Kuopio University Hospital, Kuopio, Finland

${ }^{13}$ University of Ribeirao Preto (UNAERP), Ribeirao Preto, SP, Brazil

${ }^{14}$ Center of Excellence in Animal Evolution and Genetics, Chinese Academy of Sciences, PRC

Deepa P, Chandni Halcyon Peris, Divya J Priscilla, and Ninoshka D'Souza assisted in selecting restaurants and meals and in collection and freeze drying of the foods in India. Bjorn Peters, Katja Honkanen, and Susanne Räsänen assisted in selecting restaurants, collecting meals, processing food samples, and fulfilling international shipping requirements in Finland. Obed Akwaa Harrison assisted in selecting restaurants, collecting and processing meals, and freeze drying of the foods in Ghana. Lorien Urban assisted with site instruction documents and the randomization for meal selection.

Contributors: This study was designed by SBR, SKD, JRS, JP, VS, RK, MS, AA, AT, and AK. These authors also worked with the local site authors to develop the implementation plans, and all authors except $\mathrm{KB}, \mathrm{RES}, \mathrm{CE}$, and SFT contributed to data collection. KB and RES did statistical analyses. JRS did the modeling of energy intake relative to energy requirements. SBR was responsible for the initial manuscript draft, and all authors approved the final manuscript and had full access to the data. $\mathrm{CH}$ and $\mathrm{XYZ}$ conducted the bomb calorimetry of the Chinese foods. The corresponding authors attest that all listed authors meet authorship criteria and that no others meeting the criteria have been omitted. SBR, RES, and AT are the guarantors.

Funding: This work was supported in part by the US Department of Agriculture under agreement no. 58-1950-4-003 with Tufts University and a Tufts University Provost award to SBR. The study had additional funding in Brazil from FAPESP grants 2013/18520-0 and 2013/14489-1 to VS; in China from the National Science Foundation of China grant No 91431102 to JRS and International Partnership Program of Chinese Academy of Sciences grant No GJHZ1660 to JRS; in Finland from internal funding by the University of Eastern Finland to JP; in Ghana from the University of Georgia Global Research Collaborative Grant Program to AKA. The views expressed are those of the authors. The sponsors had no role in the design, undertaking, or reporting of the study.

Competing interests: All authors have completed the ICMJE uniform disclosure form at www.icmje.org/coi_disclosure.pdf (available on request from SBR) and declare: no support from any organization for the submitted work other than that described above; no financial relationships with any organizations that might have an interest in the submitted work in the previous three years; no other relationships or activities that could appear to have influenced the submitted work.

Ethical approval: This study was deemed exempt from institutional review board approval under US federal regulation 45 CFR §46.101(b). Transparency: The guarantors affirm that the manuscript is an honest, accurate, and transparent account of the study being reported; that no important aspects of the study have been omitted; and that any discrepancies from the study as planned (and, if relevant, registered) have been explained.

Data sharing: Requests for access to data should be addressed to SBR This is an Open Access article distributed in accordance with the Creative Commons Attribution Non Commercial (CC BY-NC 4.0) license, which permits others to distribute, remix, adapt, build upon this work non-commercially, and license their derivative works on different terms, provided the original work is properly cited and the use is noncommercial. See: http://creativecommons.org/licenses/by-nc/4.0/.

1 Afshin A, Forouzanfar MH, Reitsma MB, et al, GBD 2015 Obesity Collaborators. Health Effects of Overweight and Obesity in 195 Countries over 25 Years. N Engl J Med 2017;377:13-27. doi:10.1056/NEJMoa1614362

$2 \mathrm{Ng} \mathrm{M}$, Fleming T, Robinson M, et al. Global, regional, and national prevalence of overweight and obesity in children and adults during 
1980-2013: a systematic analysis for the Global Burden of Disease Study 2013. Lancet 2014:384:766-81. doi:10.1016/S01406736(14)60460-8

3 Xu WL, Atti AR, Gatz M, Pedersen NL, Johansson B, Fratiglioni L. Midlife overweight and obesity increase late-life dementia risk: a population-based twin study. Neurology 2011;76:1568-74 doi:10.1212/WNL.0b013e3182190d09

4 Launer LJ, Harris T, Rumpel C, Madans J. Body mass index, weight change, and risk of mobility disability in middle-aged and olde women. The epidemiologic follow-up study of NHANES I. JAMA 1994;271:1093-8. doi:10.1001/jama.1994.03510380049036

5 Bertram MY, Sweeny K, Lauer JA, et al. Investing in non-communicable diseases: an estimation of the return on investment for prevention and treatment services. Lancet 2018;391:2071-8. doi:10.1016/ S0140-6736(18)30665-2

6 Tremmel M, Gerdtham U-G, Nilsson PM, Saha S. Economic Burden of Obesity: A Systematic Literature Review. Int J Environ Res Public Health 2017;14:435-53. doi:10.3390/ijerph14040435

7 Goettler A, Grosse A, Sonntag D. Productivity loss due to overweight and obesity: a systematic review of indirect costs. BMJ Open 2017;7:e014632. doi: 10.1136/bmjopen-2016-014632

8 Kudel I, Huang JC, Ganguly R. Impact of Obesity on Work Productivity in Different US Occupations: Analysis of the National Health and Wellness Survey 2014 to 2015. J Occup Environ Med 2018;60:6-11. doi:10.1097/JOM.0000000000001144

9 Kivipelto M, Ngandu T, Fratiglioni L, et al. Obesity and vascular risk factors at midlife and the risk of dementia and Alzheimer disease. Arch Neurol 2005;62:1556-60. doi:10.1001/archneur.62.10.1556

10 World Health Organization. Controlling the global obesity epidemic. 2003. http://www.who.int/nutrition/topics/obesity/en/.

11 Ritchie H, Roser M. Diet compositions. 2018. https://ourworldindata. org/diet-compositions.

12 Young LR, Nestle M. Reducing portion sizes to prevent obesity: a call to action. Am J Prev Med 2012;43:565-8. doi:10.1016/j. amepre.2012.07.024

13 De Vogli R, Kouvonen A, Gimeno D. The influence of market deregulation on fast food consumption and body mass index: a cross-national time series analysis. Bull World Health Organ 2014;92:99-107, 107A. doi:10.2471/BLT.13.120287

14 Mazidi M, Speakman JR. Higher densities of fast-food and full-service restaurants are not associated with obesity prevalence. Am J Clin Nutr 2017;106:603-13. doi:10.3945/ajcn.116.15140--7

15 Mason KE, Pearce N, Cummins S. Associations between fast food and physical activity environments and adiposity in mid-life: cross sectional, observational evidence from UK Biobank. Lancet Public Health 2018;3:e24-33. doi:10.1016/S2468-2667(17)30212-8

16 Burgoine T, Sarkar C, Webster C), Monsivais P. Examining the interaction of fast-food outlet exposure and income on diet and obesity: evidence from 51,361 UK Biobank participants. Int J Behav Nutr Phys Act 2018;15:71. doi:10.1186/s12966-018-0699-8

17 Sturm R, Hattori A. Diet and obesity in Los Angeles County 20072012: Is there a measurable effect of the 2008 "Fast-Food Ban"? Soc Sci Med 2015;133:205-11. doi:10.1016/j.socscimed.2015.03.004

18 Urban LE, Roberts SB, Fierstein JL, Gary CE, Lichtenstein AH. Temporal trends in fast-food restaurant energy, sodium, saturated fat, and trans fat content, United States, 1996-2013. Prev Chronic Dis 2014;11:E229. doi:10.5888/pcd11.140202

19 Dunford E, Webster J, Barzi F, Neal B. Nutrient content of products served by leading Australian fast food chains. Appetite 2010;55:4849. doi:10.1016/j.appet.2010.08.015

20 Urban LE, McCrory MA, Dallal GE, et al. Accuracy of stated energy contents of restaurant foods. JAMA 2011;306:287-93. doi:10.1001/ jama.2011.993

21 Urban LE, Weber JL, Heyman MB, et al. Energy Contents of Frequently Ordered Restaurant Meals and Comparison with Human Energy Requirements and U.S. Department of Agriculture Database Information: A Multisite Randomized Study. J Acad Nutr Die 2016;116:590-8.e6. doi:10.1016/j.jand.2015.11.009

22 Urban LE, Dallal GE, Robinson LM, Ausman LM, Saltzman E, Roberts SB. The accuracy of stated energy contents of reduced-energy, commercially prepared foods. J Am Diet Assoc 2010;110:116-23. doi:10.1016/j.jada.2009.10.003

23 Urban LE, Lichtenstein AH, Gary CE, et al. The energy content of restaurant foods without stated calorie information. JAMA Intern Med 2013;173:1292-9. doi:10.1001/jamainternmed.2013.6163

24 Jaworowska A, Blackham TM, Long R, et al. Nutritional Composition of Takeaway Food in the UK. Nutr Food Sci 2014;44:414-30. doi:10.1108/NFS-08-2013-0093.

25 Goffe L, Rushton S, White M, Adamson A, Adams J. Relationship between mean daily energy intake and frequency of consumption of out-of-home meals in the UK National Diet and Nutrition Survey. Int J Behav Nutr Phys Act 2017;14:131. doi:10.1186/s12966-017 0589-5

26 Nago ES, Lachat CK, Dossa RA, Kolsteren PW. Association of out-ofhome eating with anthropometric changes: a systematic review of prospective studies. Crit Rev Food Sci Nutr 2014;54:1103-16. doi:10 1080/10408398.2011.627095

27 McCrory MA, Fuss PJ, Hays NP, Vinken AG, Greenberg AS, Roberts SB. Overeating in America: association between restaurant food consumption and body fatness in healthy adult men and women ages 19 to 80. Obes Res 1999;7:564-71. doi:10.1002/j.1550-8528.1999.tb00715.x

28 Yao M, Lichtenstein AH, Roberts SB, et al. Relative influence of diet and physical activity on cardiovascular risk factors in urban Chinese adults. Int J Obes Relat Metab Disord 2003;27:920-32. doi:10.1038/sj.ijo.0802308

29 Apolzan JW, Bray GA, Hamilton MT, et al. Short-term overeating results in incomplete energy intake compensation regardless of energy density or macronutrient composition. Obesity (Silver Spring) 2014;22:119-30. doi:10.1002/oby.20587

30 Raulio S, Roos E, Rahkonen O, Prättälä R. Twenty-Year Trends of Workplace Lunches in Finland. Food Serv Technol 2005;5:57-66. doi:10.1111/j.1471-5740.2005.00112.x

31 MacLean WC, Warwick P. Food Energy: Methods of Analysis and Conversion Factors: Report of a Technical Workshop, Rome, 3-6 December 2002. Bernan Associates, 2003.

32 Karl JP, Meydani M, Barnett JB, et al. Substituting whole grains for refined grains in a 6-wk randomized trial favorably affects energybalance metrics in healthy men and postmenopausal women. Am J Clin Nutr 2017;105:589-99. doi:10.3945/ajcn.116.139683

33 The Crown. Public Health England. 2018. https://www.gov.uk/ government/organisations/public-health-england.

34 Hall KD. Did the Food Environment Cause the Obesity Epidemic? Obesity (Silver Spring) 2018;26:11-3. doi:10.1002/oby.22073

35 United States Department of Agriculture. Food expenditure series. 2018 https://www.ers.usda.gov/data-products/food-expenditures.aspx.

36 Bezerra IN, Moreira TMV, Cavalcante JB, Souza AM, Sichieri R. Food consumed outside the home in Brazil according to places of purchase. Rev Saude Publica 2017;51:15. doi:10.1590/s15188787.2017051006750

37 Tian X, Zhong L, von Cramon-Taubadel S, Tu H, Wang H. Restaurants in the Neighborhood, Eating Away from Home and BMI in China. PLoS One 2016;11:e0167721. doi:10.1371/journal.pone.0167721

38 The Neilsen Company. Dining without doing dishes: almost half globally eat away-from-home at least once a week. 2016. https:// www.nielsen.com/us/en/insights/news/2016/dining-without-doing dishes-almost-half-globally-eat-away-from-home.html.

39 Ma MG, Eating Behavior, and Culture in Chinese Society. Journal of Ethnic Foods 2015;2:195-9. doi:10.1016/j.jef.2015.11.004

40 Wang Z, Zhai F, Zhang B, Popkin BM. Trends in Chinese snacking behaviors and patterns and the social-demographic role between 1991 and 2009. Asia Pac J Clin Nutr 2012;21:253-62.

41 Kerver JM, Yang EJ, Obayashi S, Bianchi L, Song WO. Meal and snack patterns are associated with dietary intake of energy and nutrients in US adults. J Am Diet Assoc 2006;106:46-53. doi:10.1016/j. jada.2005.09.045

42 Nutrition A. Brazil: General diet/summary. 2018. http:// adoptionnutrition org/nutrition-by-country/brazil/.

43 Ovaskainen ML, Reinivuo H, Tapanainen H, Hannila ML, Korhonen T, Pakkala H. Snacks as an element of energy intake and food consumption. Eur / Clin Nutr 2006;60:494-501. doi:10.1038/ sj.ejcn.1602343

44 Fuller K. Eating habits in the Indian culture. 2018. https://classroom. synonym.com/eating-habits-in-the-indian-culture-12083298.html.

45 Nti CA. Household dietary practices and family nutritional status in rural Ghana. Nutr Res Pract 2008;2:35-40. doi:10.4162/ nrp.2008.2.1.35

46 Raynor HA. What to do about portion sizes? Roundtable discussion at the Forefronts in Portion Size Conference. Int J Obes (Lond) 2014;38(Suppl 1):S34-6. doi:10.1038/ijo.2014.87

47 Almiron-Roig E, Palla L, Guest K, et al. Factors that determine energy compensation: a systematic review of preload studies. Nutr Rev 2013;71:458-73. doi:10.1111/nure.12048

48 Power ML, Schulkin J. Anticipatory physiological regulation in feeding biology: cephalic phase responses. Appetite 2008;50:194-206. doi:10.1016/j.appet.2007.10.006

49 Lasschuijt MP, Mars M, de Graaf C, Smeets PAM. Exacting Responses: Lack of Endocrine Cephalic Phase Responses Upon Oro-Sensory Exposure. Front Endocrinol (Lausanne) 2018;9:332. doi:10.3389/ fendo.2018.00332

50 English L, Lasschuijt M, Keller KL. Mechanisms of the portion size effect. What is known and where do we go from here? Appetite 2015;88:39-49. doi:10.1016/j.appet.2014.11.004

51 Bleich SN, Wolfson JA, Jarlenski MP, Block JP. Restaurants With Calories Displayed On Menus Had Lower Calorie Counts Compared To Restaurants Without Such Labels. Health Aff (Millwood) 2015;34:1877-84. doi:10.1377/hlthaff.2015.0512

52 Roberts S, Ubran LE, Gilhooly CH. Calculating calories and nutrients in meals. 2018. https://hnrca.tufts.edu/flipbook/resources/restaurantmeal-calculator. 
53 Gase L, Dunning L, Kuo T, Simon P, Fielding JE. Restaurant owners perspectives on a voluntary program to recognize restaurants for offering reduced-size portions, Los Angeles County, 2012. Prev Chronic Dis 2014;11:E44. doi:10.5888/pcd11.130310

54 Wang Y, Wang L, Xue H, Qu W. A Review of the Growth of the Fast Food Industry in China and Its Potential Impact on Obesity. Int J Environ Res Public Health 2016;13:1112. doi:10.3390/ ijerph13111112

55 National Institute for Health and Welfare. National FinHealth Study. 2018. https://thl.fi/fi/web/thlfi-en/research-and-expertwork/ population-studies/national-finhealth-study.
56 Roos E, Sarlio-Lähteenkorva S, Lallukka T. Having lunch at a staff canteen is associated with recommended food habits. Public Health Nutr 2004;7:53-61. doi:10.1079/PHN2003511

57 Arora S, Nanda K. What's cooking with Indian diners. 2017. https:// www.nielsen.com/in/en/insights/reports/2017/whats-cooking-withindian-diners.html.

58 Maheshwari A, Ghosh S, Marwah D, et al. India's Food Service Industry: Growth Recipe. KPMG International: Consumer Markets, 2016.

Supplementary tables and figures 